\title{
Conhecimento entre acadêmicos sobre doação de corpos para o ensino de Anatomia
}

\section{Humana}

\author{
Knowledge between academics about body donation for the teaching of Human Anatomy \\ Conocimiento entre académicos sobre la donación de cuerpos para la enseñanza de Anatomía
}

\section{Humana}

Recebido: 11/02/2021 | Revisado: 16/02/2021 | Aceito: 19/02/2021 | Publicado: 28/02/2021

Rariane Bernardino Marani

ORCID: https://orcid.org/0000-0001-5978-2025

Centro Universitário de Maringá, Brasil

E-mail: rarimarani@gmail.com

Catchia Hermes-Uliana

ORCID: https://orcid.org/0000-0002-2698-0022

Universidade Federal de Mato Grosso do Sul, Brasil

E-mail: catchiahermes@ hotmail.com

Ana Paula da Silva

ORCID: https://orcid.org/0000-0003-4163-4977

Universidade Federal de Ciências da Saúde de Porto Alegre, Brasil

E-mail: paulasilvabio@gmail.com

Elizangela dos Anjos Silva

ORCID: https://orcid.org/0000-0001-9470-1948

Universidade Federal do Pampa, Brasil

E-mail: elizanjosfisio@gmail.com

Heber Amilcar Martins

ORCID: https://orcid.org/0000-0003-1808-8138

Centro Universitário de Maringá, Brasil

E-mail: heberamartins@gmail.com

\begin{abstract}
Resumo
A utilização de cadáveres humanos para fins de ensino é uma prática comum em todo o mundo. Objetivou-se relatar o conhecimento dos acadêmicos sobre a doação de cadáveres para o ensino de anatomia humana. Para tanto 200 acadêmicos das áreas de ciências biológicas e da saúde (CBS), ciências exatas (CE), ciências tecnológicas e agrárias (CTA), e ciências humanas e sociais (CHS) responderam um instrumento elaborado pelos autores. Os participantes do sexo masculino se mostraram mais propensos a doarem o seu corpo ou de um familiar $(36,7 \%$ e $56,9 \%$ respectivamente). O conhecimento sobre a utilização $(98,0 \%)$ e como os cadáveres são utilizados $(70,6 \%)$ em atividades de ensino $(98,0 \%)$, bem como a concordância em utilizar este recurso $(96,1 \%)$ foram predominantes entre os acadêmicos das CBS. Os resultados demonstram um consenso sobre a importância do uso de cadáveres no ensino, pois a maioria dos acadêmicos concordam com o ensino em cadáveres e seu uso no desenvolvimento de pesquisas médicas científicas. O maior conhecimento a respeito da preservação, manipulação e importância do cadáver para a formação técnica e humanizada está entre os acadêmicos das CBS. Apesar disso, a maioria dos acadêmicos manifestou o desejo de não doar seus corpos.
\end{abstract}

Palavras-chave: Anatomia; Cadáver; Corpo humano; Restos mortais.

\begin{abstract}
The use of human corpses for teaching purposes is a common practice worldwide. The objective was to report the knowledge of academics on the donation of cadavers for the teaching of human anatomy. For that purpose 200 academics in the areas of biological sciences and health (CBS), exact sciences (CE), technological and agrarian sciences (CTA), and human and social sciences (CHS) answered an instrument developed by the authors. Male participants were more likely to donate their body or that of a family member (36.7\% and $56.9 \%$ respectively). Knowledge about the use (98.0\%) and how corpses are used (70.6\%) in teaching activities $(98.0 \%)$, as well as the agreement to use this resource $(96.1 \%)$ were predominant among CBS academics. The results demonstrate a consensus on the importance of using cadavers in teaching, as most academics agree with teaching on cadavers and their use in the development of scientific medical research. The greatest knowledge regarding the preservation, manipulation and importance of the corpse for technical and humanized training is among CBS academics. Despite this, most academics expressed a desire not to donate their bodies.
\end{abstract}

Keywords: Anatomy; Corpse; Human body; Mortal remains. 


\section{Resumen}

El uso de cadáveres humanos con fines didácticos es una práctica común en todo el mundo. El objetivo fue informar sobre los conocimientos de los académicos sobre la donación de cadáveres para la enseñanza de la anatomía humana. Para ello, 200 académicos de las áreas de ciencias biológicas y de la salud (CBS), ciencias exactas (CE), ciencias tecnológicas y agrarias (CTA) y ciencias humanas y sociales (CHS) respondieron a un instrumento elaborado por los autores. Los participantes masculinos tenían más probabilidades de donar su cuerpo o el de un miembro de la familia (36,7\% y 56,9\% respectivamente). El conocimiento sobre el uso $(98.0 \%)$ y cómo se usan los cadáveres $(70.6 \%)$ en las actividades docentes (98.0\%), así como el acuerdo para usar este recurso (96.1\%) fueron predominantes entre los académicos de CBS. Los resultados demuestran un consenso sobre la importancia del uso de cadáveres en la docencia, ya que la mayoría de los académicos están de acuerdo con la enseñanza sobre cadáveres y su uso en el desarrollo de la investigación médica científica. El mayor conocimiento sobre la preservación, manipulación e importancia del cadáver para la formación técnica y humanizada se encuentra entre los académicos de CBS. A pesar de esto, la mayoría de los académicos expresaron su deseo de no donar sus cuerpos.

Palabras clave: Anatomía; Cadáver; Cuerpo humano; Restos mortales.

\section{Introdução}

A utilização de cadáveres humanos para fins de ensino é uma prática comum em todo o mundo. O uso de cadáveres para o ensino da anatomia humana é relatado desde o período medieval, que iniciou com o uso de animais. Posteriormente, foi iniciada a prática com corpos humanos, onde utilizavam pessoas que estavam presas ou condenadas à morte (Mitchell et al., 2011).

A utilização de cadáveres é de alta importância para o ensino da anatomia, apesar da relevância do estudo em peças cadavéricas reais, a doação de corpos para tais fins apresenta-se baixa em todo o mundo. A escassez de material cadavérico está atrelada a dificuldade de captação e distribuição de cadáveres, e as razões dessa dificuldade devem ser devidamente perscrutadas, a fim de que possam ser sanadas (Cordeiro \& Menezes, 2019; Volanek \& Rissi, 2019).

Assim, a doação de corpos é um dos meios mais importantes para obtenção de cadáveres em várias universidades ao redor do mundo (da Rocha et al., 2017). A oferta de cadáveres diminuiu, pois, a falta de informações atinge tanto os possíveis doadores, como os Serviços de Verificação de Óbitos (SVO). Ainda, questões psicológicas envolvendo fatores culturais, religiosos e emocionais podem estar diretamente relacionadas com a doação de corpos. Portanto, há programas de doação de corpos que conscientizam a importância do ato e o esclarecimento do uso para fins acadêmicos, junto à sociedade jurídica e civil, através de intervenções como, realização de Cerimônias em Homenagem aos doadores e, também projetos de pesquisa com eventos, tais como Museus de Anatomia (da Silva Baptista et al., 2012; Rocha, Tormes, Lehmann, Schwab \& Canto, 2013)

A disciplina de anatomia humana fornece suporte para a correlação entre localização, sintopia e função de cada parte do corpo em uma visão tridimensional, adicionada à interação entre o ensino básico e os conhecimentos clínicos. Para as aulas, além das peças anatômicas humanas são utilizados modelos anatômicos sintéticos e outros materiais didáticos, como atlas, softwares e materiais disponíveis na internet (Costa, Costa \& Lins, 2012; da Rocha et al., 2017). A fim de trazer novas formas e alternativas de ensino a disciplina, vem-se estudando novos meios que possam contribuir para o seu ensino aprendizagem (Colares, Vidotti \& Sant’Ana, 2019). No entanto, a utilização de cadáveres (dissecação e prossecção) ainda é um componente essencial no processo de ensino de anatomia (Melo \& Pinheiro, 2010).

A anatomia continua a ser uma das bases da formação médica, e por isso o corpo humano morto continua a desempenhar um papel central na aquisição de conhecimentos e no progresso científico. O manuseio de cadáveres ajuda na humanização dos profissionais e no contexto bioético, no qual confirma não ser apenas objeto de estudo, mas um meio de agregar habilidades técnicas, conceitos de ética e humanização. É necessário ter maturidade emocional frente ao cadáver, que contribui no desenvolvimento e na preparação do equilíbrio emocional frente à morte. No entanto, a ausência do uso de cadáveres dificulta o processo de humanização e a relação médico paciente, uma vez que o processo da morte está relacionado 
à medicina e o diálogo sobre tal fato ajuda na construção do conhecimento médico (Costa et al., 2012).

Considerando que o corpo humano é um material extremamente necessário para o estudo da prática anatômica, e que a sua doação se encontra em escassez, comprometendo a qualidade na formação de dos futuros profissionais da área de saúde, objetivou-se analisar o conhecimento entre acadêmicos do ensino superior sobre a doação de corpos para o ensino da anatomia humana. Dessa forma, esse trabalho pode contribuir na divulgação da possibilidade de doação de corpo para o ensino e pesquisa na área da anatomia humana.

\section{Metodologia}

\subsection{Delineamento da pesquisa}

Essa pesquisa apresentou análise quantitativa e qualitativa composta de descrição e exploração (Pereira, et al., 2018) por meio da aplicação de instrumento adaptado de Abbasi Asl et al. (2017) que permitiu buscar dados dos acadêmicos de diferentes áreas de conhecimento sobre doação de corpos para o estudo da disciplina de anatomia humana. O método envolveu o aprimoramento de ideias, a busca de informações sobre a temática em material didático, análise e interpretação dos dados coletados a partir de um questionário aplicado.

Foram coletadas informações de 200 acadêmicos de quatro áreas de conhecimento de uma instituição de ensino superior de Maringá/PR que aceitaram participar do estudo. A coleta de dados foi realizada por equipe treinada e ocorreu em sala de aula, após autorização do professor que ministrava aula no momento. Todos os estudantes presentes foram convidados a participar. As áreas foram nomeadas de acordo com a nomenclatura definida pela instituição: 51 respondentes eram da área de ciências biológicas e da saúde (CBS), 50 da área de ciências exatas (CE), 49 da área de ciências humanas e sociais aplicadas (CHS), e 50 da área de ciências tecnológicas e agrárias (CTA).

As orientações quanto aos objetivos da pesquisa, o caráter sigiloso das informações coletadas, o direito de recusar-se de participar em qualquer fase de execução, assim como a garantia de não sofrer qualquer ônus ou gratificação pela sua participação e sobre o preenchimento do instrumento, foram devidamente passadas aos acadêmicos.

Os critérios de inclusão foram acadêmicos de ambos os sexos, estar presente no dia proposto para a coleta de dados, maiores de 18 anos, aceitar participar do estudo e assinar o Termo de Consentimento Livre e Esclarecido (TCLE). Excluíramse aqueles com idade inferior a 18 anos e que não manifestaram interesse em participar da pesquisa.

Essa pesquisa foi submetida ao Comitê de Ética e Pesquisa (CEP) para prévia avaliação, amparado pelas Resoluções nos.466/2012 e 510/2016 do Conselho Nacional de Saúde (CNS), no qual obteve a aprovação com o parecer de número 011574/2018. Aos entrevistados desta pesquisa foram solicitados a assinarem o TCLE em duas vias, permanecendo uma com o participante e outra com a pesquisadora. A aplicação do instrumento transcorreu nos períodos diurno e noturno, no período entre junho e julho de 2018.

\subsection{Instrumento}

O instrumento foi elaborado pelos autores após uma extensa revisão da literatura e identificação dos pontos chave, as questões abordavam dados sociodemográficos como sexo, idade, religião área do conhecimento e semestre. Além de questões sobre intensão de ser doador ou autorizar a doação de familiares, Frequência quanto ao ensino em cadáveres, possibilidade de realizar doações de algum parente pós-morte, o uso de cadáveres no auxílio de desenvolvimento de pesquisas médicascientificas e à preservação dos cadáveres, sua preparação e uso para fins didáticos e seu tratamento.

\subsection{Tratamento estatístico}

Os dados coletados foram plotados em uma planilha do Excel (Microsoft Corporation®, versão 2016, Redmond, 
WA). Posteriormente foram exportados para o Programa Estatístico SPSS (Statistical Package for the Social Sciences, Version 23.0, Armonk, NY: IBM Corp.). Os resultados foram apresentados como distribuição de frequência. As associações das variáveis foram analisadas pelos testes do Chi-square e de Fisher, com 95\% de intervalo de confiança e nível de significância de $5 \%$.

\section{Resultados}

Foram aplicados 200 instrumentos nos diferentes cursos de graduação e diferentes áreas de conhecimento, CBS, CE, CHS e CTA. Quanto ao semestre, 57 (28,5\%) alunos pertenciam ao primeiro semestre, $116(58 \%)$ ao terceiro semestre e 27 $(13,5 \%)$ estavam no sétimo semestre. Com relação ao sexo, 91 (45,5\%) participantes eram do sexo feminino e 109 (54,5\%) do sexo masculino. Referente à religião, 127 (63,5\%) eram católicos, 33 (18,9\%) evangélicos, 6 (3\%) espíritas, 3 (1,5\%) ateus, 3 $(1,5 \%)$ agnósticos e 25 (12,5\%) sem religião (Tabela 1$)$.

Tabela 1. Frequência de distribuição dos acadêmicos entrevistados por área do conhecimento: ciências biológicas e da saúde (CBS), ciências exatas (CE), ciências humanas e sociais (CHS) e ciências tecnológicas e agrárias (CTA); semestre; sexo e religião.

\begin{tabular}{cccc}
\hline Característica & & N & Frequência (\%) \\
\hline \multirow{3}{*}{ Área do conhecimento } & CBS & 51 & 25,5 \\
& CE & 50 & 25,0 \\
& CHS & 49 & 24,5 \\
& CTA & 50 & 25,0 \\
\hline \multirow{3}{*}{ Semestre } & Primeiro & 57 & 28,5 \\
& Terceiro & 116 & 58,0 \\
& Sétimo & 27 & 13,5 \\
\hline \multirow{2}{*}{ Sexo } & Feminino & 91 & 45,5 \\
& Masculino & 109 & 54,5 \\
\hline \multirow{3}{*}{ Religião } & Católico & 127 & 63,5 \\
& Evangélico & 33 & 18,9 \\
& Espírita & 6 & 3,0 \\
& Ateu & 3 & 1,5 \\
& Agnóstico & 3 & 1,5 \\
& Sem religião & 25 & 12,5 \\
\hline
\end{tabular}

Fonte: Autores.

Para comparar as áreas de estudo com as religiões, para fins estatísticos, as religiões foram separadas em dois grupos: a) católicos, evangélicos e espíritas; e b) agnósticos, ateus e sem religião. A área de conhecimento que apresentou maior número de pessoas dentro do grupo de católicos, evangélicos e espíritas foi a área de CTA com 47 (94,0\%) dos participantes. Esse resultado foi significativo quando comparado com as demais áreas, CBS com 46 (90,2\%), CE 39 (78,0\%) e CHS 37 $(75,5 \%)(\mathrm{p}<0,05 ;$ Tabela 2). Na área de CHS, o número de pessoas dentro do grupo de agnósticos, ateus e sem religião foi maior $(12 ; 24,5 \%)$ quando comparado com as outras áreas, CBS com $5(8,8 \%)$, CE $11(22,0 \%)$ e CTA $3(6,0 \%)$ (p< 0,05; Tabela 2). 
Tabela 2. Distribuição da frequência em relação a religião (católico, evangélico e espírita e agnóstico, ateu ou sem religião) de acordo com a área do conhecimento: ciências biológicas e da saúde (CBS), ciências exatas (CE), ciências humanas e sociais (CHS) e ciências tecnológicas e agrárias (CTA).

\begin{tabular}{|c|c|c|c|}
\hline & \multirow[b]{2}{*}{ Característica } & \multicolumn{2}{|c|}{ Religião } \\
\hline & & $\begin{array}{c}\text { Católico, evangélico, } \\
\text { espírita }\end{array}$ & $\begin{array}{c}\text { Agnóstico, ateu, sem } \\
\text { religião }\end{array}$ \\
\hline & CBS & $46(90,2 \%)$ & $5(8,8 \%)$ \\
\hline Área do & $\mathrm{CE}$ & $39(78,0 \%)$ & $11(22,0 \%)$ \\
\hline \multirow[t]{2}{*}{ conhecimento } & CHS & $37(75,5 \%)$ & $12(24,5 \%)^{*}$ \\
\hline & CTA & $47(94,0 \%)^{*}$ & $3(6,0 \%)$ \\
\hline
\end{tabular}

* Houve diferença significante entre a religião quando comparadas as diferentes áreas, de acordo com o teste Chi-Square ( $\mathrm{p}=0,025)$. Fonte: Autores.

Quanto à possibilidade de serem doadores, os participantes do sexo masculino $(40 ; 36,7 \%)$ apresentaram maior adesão à doação quando comparados ao sexo feminino $(19 ; 20,9 \%)(\mathrm{p}<0,05$; Tabela 3$)$. Sobre a possibilidade de autorizar a doação do corpo de um parente, novamente houve maior adesão entre o sexo masculino $(62 ; 56,9 \%)$ em detrimento do sexo feminino $(35$; $38,5 \%),(\mathrm{p}<0,05 ;$ Tabela 3).

Tabela 3. Frequência quanto à intensão de ser doador ou autorizar a doação de familiares por sexo entre os acadêmicos entrevistados.

Se você fosse responsável pela

Característica

Seria um doador?

doação voluntária de algum

parente pós-morte, autorizaria?

\begin{tabular}{|c|c|c|c|c|c|}
\hline & & \\
\hline & & Sim & Não & Sim & Não \\
\hline \multirow{2}{*}{ Sexo } & Feminino & $19(20,9 \%)^{*}$ & $72(79,1 \%)$ & $35(38,5 \%)^{*}$ & $56(61,5 \%)$ \\
\hline & Masculino & $40(36,7 \%)^{*}$ & $69(63,3 \%)$ & $62(56,9 \%)^{*}$ & $47(43,1 \%)$ \\
\hline
\end{tabular}

* Houve diferença significante entre a possibilidade de ser um doador e os sexos, de acordo com o teste Chi-Square $(p=0,015)$ e quanto a responsabilidade pela doação voluntária de um parente pós-morte, de acordo com o teste Chi- Square (p=0,009).

Fonte: Autores.

Quando perguntados sobre o fato de concordarem com o ensino/estudo em cadáveres a maioria dos acadêmicos respondeu que concorda, sendo observado diferença significante entre a área de CBS (49; 96,1\%), comparado às demais áreas, CE e CHS $(41 ; 82,0 \%)$ e CTA $(35 ; 70,0 \%)$ (p< 0,05; Tabela 4). A maior proporção de entrevistados que não concordaram com o ensino/estudo em cadáveres foi observada em CTA $(3 ; 6,0 \%)$ participantes, seguida pela área de CBS com 1 (2,0\%) participante $(\mathrm{p}<0,05$; Tabela 4$)$.

Em relação à área do conhecimento, quanto à possibilidade de realizar a doação do corpo de algum parente pós-morte, houve diferença significativa em CBS, onde 24 (47,1\%) dos 51 (100\%) entrevistados concordaram com tal ato, comparado com as demais áreas, a concordância foi de 21 (42,0\%) entrevistados em CE, 12 (24,5\%) em CHS e 11 (22,0\%) em CTA (p< 0,05; Tabela 4).

Ao serem questionados sobre a possibilidade do uso de cadáveres ajudar no desenvolvimento de pesquisas médicascientificas, houve diferença significativa na área de CE, em que 49 (98,0\%) dos 50 (100\%) entrevistados concordaram, em relação às demais áreas, CBS (48; 94,1\%), CHS (43; 87,8\%) e CTA (40; 80,0\%) (p< 0,05; Tabela 4). 
Ainda sobre a concordância ou não do uso de cadáveres na ajuda de pesquisas médicas-cientificas, foi observado predomínio na resposta "não concordo e nem discordo" nos entrevistados de CTA $(10 ; 20,0 \%)$ quando comparado às áreas CHS $(6 ; 12,2 \%)$, CBS $(2 ; 3,9 \%)$ e CE $(1 ; 2,0 \%)(\mathrm{p}<0,05 ;$ Tabela 4$)$.

Tabela 4. Frequência quanto ao ensino em cadáveres, possibilidade de realizar doações de algum parente pós-morte e o uso de cadáveres no auxílio de desenvolvimento de pesquisas médicas-cientificas de acordo com as áreas do conhecimento: ciências biológicas e da saúde (CBS), ciências exatas (CE), ciências humanas e sociais (CHS) e ciências tecnológicas e agrárias (CTA).

\section{Área do conhecimento}

\section{Característica}

CBS CE CHS CTA

\begin{tabular}{|c|c|c|c|c|c|}
\hline \multirow{3}{*}{$\begin{array}{l}\text { Concorda com o } \\
\text { ensino/ estudo em } \\
\text { cadáveres? }\end{array}$} & Concorda & $49(96,1 \%)^{*}$ & $41(82,0 \%)$ & $41(83,7 \%)$ & $35(70,0 \%)$ \\
\hline & $\begin{array}{l}\text { Não concordo } \\
\text { nem discordo }\end{array}$ & $1(2,0 \%)$ & $9(18,0 \%)$ & $8(16,3 \%)$ & $12(24,0 \%)$ \\
\hline & Não concordo & $1(2,0 \%)$ & $0(0,0 \%)$ & $0(0,0 \%)$ & $3(6,0 \%)^{*}$ \\
\hline Concorda com a & Concorda & $24(47,1 \%)^{*}$ & $21(42,0 \%)$ & $12(24,5 \%)$ & $11(22,0 \%)$ \\
\hline $\begin{array}{l}\text { possibilidade de } \\
\text { realizar a doação de } \\
\text { um corpo de algum }\end{array}$ & $\begin{array}{l}\text { Não concordo } \\
\text { nem discordo }\end{array}$ & $17(33,3 \%)$ & $22(44,0 \%)$ & $21(42,9 \%)$ & $25(50,0 \%)$ \\
\hline parente pós-morte? & Não concordo & $10(9,6 \%)$ & $7(14,0 \%)$ & $16(32,7 \%)$ & $14(28,0 \%)$ \\
\hline Concorda que o uso & Concorda & $48(94,1 \%)$ & $49(98,0 \%)^{*}$ & $43(87,8 \%)$ & $40(80,0 \%)$ \\
\hline $\begin{array}{c}\text { de cadáveres ajuda } \\
\text { no desenvolvimento } \\
\text { de pesquisas }\end{array}$ & $\begin{array}{l}\text { Não concordo } \\
\text { nem discordo }\end{array}$ & $2(3,9 \%)$ & $1(2,0 \%)$ & $6(12,2 \%)$ & $10(20,0 \%)^{*}$ \\
\hline médicas-cientificas? & Não concordo & $1(2,0 \%)$ & $0(0,0 \%)$ & $0(0,0 \%)$ & $0(0,0 \%)$ \\
\hline
\end{tabular}

* Houve diferença significante entre as áreas quanto ao uso estudo de cadáveres, de acordo com o teste Chi-Square ( $\mathrm{p}=0,010$ ), quanto a possibilidade de realizar a doação de um corpo de algum parente pós-morte, de acordo com o teste Chi-Square ( $\mathrm{p}=0$,042), e quanto ao uso de cadáveres no desenvolvimento de pesquisas médicas-cientificas, de acordo com o teste Chi-Square ( $\mathrm{p}=0,022)$. Fonte: Autores.

Quanto ao conhecimento sobre a preservação dos cadáveres predominou o conhecimento da área de CBS (45; 88,2\%), seguido de CTA $(22 ; 44,0 \%)$, CE $(20 ; 40,0 \%)$ e CHS $(19 ; 38,8 \%)$ (p< 0,05; Tabela 5). Em relação ao conhecimento a respeito do tempo decorrido desde a morte até a preparação do cadáver para fins didáticos houve predominância da área de CBS (18; $35,3 \%)$, seguindo de CHS $(2 ; 4,1 \%)$ e CTA $(2 ; 4,0 \%)$ e CE $(1 ; 2,0 \%)$ (p<0,05; Tabela 5). Dentre as áreas, a que apresentou maior conhecimento sobre o uso de cadáveres para fins didáticos foi CBS (46; 90,2\%), seguido de CE (33; 66,0\%) e CTA (33; 66,0\%) e CHS (29; 59,2\%) (p<0,05; Tabela 5). Questionados sobre a maneira com que os cadáveres são manipulados, os entrevistados de CBS foram os que afirmaram ter maior conhecimento, com $36(70,6 \%)$ participantes, seguido de 12 (24,0\%) de CTA, 10 (20,0\%) de CE e10 (20,4\%) CHS (p< 0,05; Tabela 5). 
Tabela 5. Frequência quanto à preservação dos cadáveres, sua preparação e uso para fins didáticos, e seu tratamento de acordo com as áreas do conhecimento: ciências biológicas e da saúde (CBS), ciências exatas (CE), ciências humanas e sociais (CHS) e ciências tecnológicas e agrárias (CTA).

\begin{tabular}{|c|c|c|c|c|c|}
\hline \multirow{2}{*}{ Característica } & & \multicolumn{4}{|c|}{ Área do conhecimento } \\
\hline & & CBS & $\mathbf{C E}$ & CHS & CTA \\
\hline \multirow{2}{*}{$\begin{array}{l}\text { Conhece a maneira com que } \\
\text { os cadáveres são preservados? }\end{array}$} & Conhece & $45(88,2 \%)^{*}$ & $20(40,0 \%)$ & $19(38,8 \%)$ & $22(44,0 \%)$ \\
\hline & $\begin{array}{l}\text { Não } \\
\text { conhece }\end{array}$ & $6(11,8 \%)$ & $30(60,0 \%)$ & $30(61,2 \%)$ & $28(56,0 \%)$ \\
\hline \multirow{2}{*}{$\begin{array}{l}\text { Conhece o tempo decorrido } \\
\text { desde a morte até a preparação } \\
\text { do cadáver para fins didáticos? }\end{array}$} & Conhece & $18(35,3 \%)^{*}$ & $1(2,0 \%)$ & $2(4,1 \%)$ & $2(4,0 \%)$ \\
\hline & $\begin{array}{l}\text { Não } \\
\text { conhece }\end{array}$ & $33(64,7 \%)$ & $49(98,0 \%)$ & $47(95,9 \%)$ & $48(96,0 \%)$ \\
\hline \multirow{2}{*}{$\begin{array}{c}\text { Conhece o uso de cadáveres } \\
\text { para fins didáticos? }\end{array}$} & Conhece & $46(90,2 \%)^{*}$ & $33(66,0 \%)$ & $29(59,2 \%)$ & $33(66,0 \%)$ \\
\hline & $\begin{array}{l}\text { Não } \\
\text { conhece }\end{array}$ & $5(9,8 \%)$ & $17(34,0 \%)$ & $20(40,8 \%)$ & $17(34,0 \%)$ \\
\hline \multirow{2}{*}{$\begin{array}{l}\text { Conhece como os cadáveres } \\
\text { são tratados/ manipulados? }\end{array}$} & Conhece & $36(70,6 \%)^{*}$ & $10(20,0 \%)$ & $10(20,4 \%)$ & $12(24,0 \%)$ \\
\hline & $\begin{array}{l}\text { Não } \\
\text { conhece }\end{array}$ & $15(29,4 \%)$ & $40(80,0 \%)$ & $39(79,6 \%)$ & $38(76,0 \%)$ \\
\hline
\end{tabular}

* Houve diferença significante entre as áreas, de acordo com o teste Chi-Square $(\mathrm{p}=0,000)$. Fonte: Autores.

\section{Discussão}

Este estudo buscou analisar o conhecimento entre os acadêmicos do nível superior de ensino a respeito da doação de corpos para o ensino de anatomia humana. Além disso, foram avaliadas questões religiosas, sexo, o uso de cadáveres para o ensino da anatomia humana (sua manipulação, preparo e preservação) e seu papel no desenvolvimento de pesquisas médicascientificas visto que, tais variáveis podem afetar diretamente a ato da doação.

Ao comparar as áreas de estudo com as religiões, temos que a predominância entre os católicos, evangélicos e espiritas, entretanto os agnósticos, ateu e sem religião se fazem presente. Tal identidade religiosa pode estar relacionada com o fato de o Brasil ser um país com ampla variedade religiosa, embora, predomine o catolicismo, recebe influências de outras culturas que interferem nas decisões quanto às praticadas com o corpo, como afirma Queiroz (2005). Além disso, por ser um país majoritariamente católico, apenas $4 \%$ dos doadores são ateus. É válido ressaltar que entre os não católicos, os adeptos do budismo e do espiritismo incentivam a doação, visto que para eles, o corpo é uma parte ínfima de sua existência, podendo ser deixado para estudos (da Rocha et al., 2017).

Neste trabalho temos o perfil de doador sendo masculino, um perfil diferente foi encontrado por Rocha et al. (2017), representado por: mulher, branca, por volta dos 60 anos, solteira, pertencente a um grupo religioso, com ensino médio completo e pertencente a classe média. Além disso, a maioria dos acadêmicos manifestou o desejo de não doar seus corpos em ambos os sexos, a baixa aptidão para doar o próprio corpo cadavérico condiz com a atual escassez de material anatômico nas instituições de ensino. Mwachaka, Mandela e Saidi (2016), também evidenciou que a maioria dos acadêmicos não estava disposta a doar seu corpo, as justificativas pela negativa de doação incluíram desde barreiras religiosas até o descaso visto na utilização das peças. Outro fator que deve ser levado em consideração é a idade dos entrevistados no presente estudo, que tiveram em torno de 20 anos, nessa idade dificilmente os acadêmicos estão pensando sobre a morte, algo que parece ser mais 
influente quando se envelhece.

Em relação à possibilidade de autorizar a doação do corpo de um parente, quando comparamos por sexo, houve uma predominância masculina e, quando comparamos por área de conhecimento, CBS apresentou maior porcentagem na possibilidade de realizar a doção do corpo de algum parente pós-morte. Mwachaka et al. (2016), demonstrou que a maioria dos acadêmicos recomenda a doação de corpos, visto que a dissecação, de acordo com os entrevistados, era a melhor maneira de aprender anatomia. Mostrando que, apesar dos entrevistados não estarem dispostos a doar seus próprios corpos, recomendariam a doação para outras pessoas.

Vale destacar que concordar com a possibilidade de uma doação não remete ao ato de doar, conforme apresentado no estudo de Abbasi Asl et al. (2017), onde a maioria dos entrevistados concordou com a doação, no entanto menos de $30 \%$ doaria o próprio corpo. Além disso, a ausência e/ou limitação de informações sobre a doação de cadáveres contribui para o número restrito de doadores. Para Quiroga-Garza et al. (2017), a informação sobre o processo de doação é importante principalmente para quem se mostra indeciso, uma vez que sua pesquisa foi realizada em duas etapas, na qual comparou as respostas diante da mesma pergunta, obtendo um número muito menor de indecisos na segunda aplicação e um número maior de adeptos tanto a doar seus próprios corpos, quanto ao de seus familiares. Dembogurski et al. (2011), observaram que a maior disponibilidade de informações sobre o processo de doação elevou o número de doações efetivas em uma instituição no qual um programa de doação de corpos foi implantado.

Apesar do desejo de não doar seus corpos a maioria dos acadêmicos concordou com o estudo em cadáveres. $\mathrm{O}$ uso de cadáveres para o ensino/estudo obteve maior concordância na área de CBS, o que mostra a importância da temática da morte na formação da área, sendo que o cadáver não pode ser visto apenas como uma peça de estudo, pois possui vínculos em vida em que a discussão sobre o assunto promove a melhor maneira possível de se lidar com alguns aspectos da vida e da morte (Cintra, 2017). Em contraponto, acadêmicos das demais áreas do conhecimento podem apresentar maior dificuldade para compreender a importância da utilização de cadáveres no processo de ensino-aprendizagem. Medeiros et al. (2013), demonstram que o contato dos acadêmicos com peças anatômicas biológicas promove o desenvolvimento de habilidades específicas e psicossociais que tornam o aprendizado mais efetivo e reflexivo, aproximando o binômio aluno-professor, intensificando a troca de experiências e consolidação do conhecimento anatômico.

Sobre a possibilidade do uso de cadáveres ajudar no desenvolvimento de pesquisas médicas-cientificas, a maioria dos acadêmicos independente da área respondeu que concorda. Portugal et al. (2011), compararam a eficácia do aprendizado como emprego de modelos anatômicos sintéticos e material cadavérico no estudo da pelve feminina, e observaram que o modelo sintético não seria suficiente para esclarecer o tema, mas um complemento às peças naturais. Além disso, afirmaram que apenas a aula teórica não seria suficiente, sendo indispensável à aula prática. Costa et al. (2012) por meio de um questionário concluíram que 88,9\% dos acadêmicos consideravam o uso de cadáveres humanos indispensável nas aulas de anatomia, além de $95,7 \%$ dos mesmos não concordarem com o uso de cadáveres exclusivamente para pesquisas científicas, visto que $81,9 \%$ dos entrevistados acreditam não ser possível a substituição dos cadáveres por programas de computadores e materiais sintéticos.

O tempo decorrido desde a morte até a preparação do cadáver para fins didáticos apresenta-se ainda desconhecido, visto que a maior porcentagem alcançada foi de 35,3\% em CBS. Essa predominância pode estar relacionada com a presença de aulas introdutórias explicando a importância do uso de cadáveres e sobre o processo de doação, no qual quando comparado a doação voluntária é menos complexo quando comparado a doação de corpos não reclamados, uma vez que neste caso, há a necessidade de publicidade legal, com pelo menos 10 inserções nos principais meios de comunicação no período de 30 dias, com o objetivo de encontrar pessoas interessadas em reclamar o corpo. Após este período, o cadáver poderá ser destinado à doação para fins acadêmicos, exceto quando há indícios de que a morte tenha resultado de ação criminosa (Portugal et al., 
2011).

Todas as áreas apresentaram sua maioria como conhecedoras do sobre o uso de cadáveres para fins didáticos. Considerando que os acadêmicos das áreas da saúde e biológicas têm contato frequente com material biológico nos laboratórios de anatomia humana, o conhecimento sobre as rotinas do uso de cadáveres é mais difundido. Resultados semelhantes foram descritos por Costa et al (2012), em que maioria dos acadêmicos afirmaram ter conhecimento sobre manipulação de cadáveres. Entretanto, quando a maneira com que os cadáveres são manipulados, apenas CBS apresentou a maioria como conhecedoras, provavelmente porque os acadêmicos da CBS possuem mais informações sobre o assunto e compartilham intelectualmente da necessidade de material anatômico nos laboratórios de ensino, visando contribuir para a formação dos futuros profissionais da saúde.

\section{Conclusão}

Há um consenso de que a utilização de cadáveres para o ensino de anatomia humana é de extrema importância. Dessa forma, a doação voluntária de corpo proporciona maior disponibilidade de cadáveres para atividades de ensino e pesquisa.

Independente da área do conhecimento, a maioria dos acadêmicos concordam com o estudo em cadáveres e de seu uso no desenvolvimento de pesquisas médicas científicas, apesar de concordarem com a importância da utilização de cadáveres a maioria não doaria seu próprio corpo, esse fator colabora com a escassez de material cadavérico nas instituições de ensino superior.

O maior conhecimento a respeito da preservação, manipulação e importância do cadáver para a formação técnica e humanizada está entre os acadêmicos das CBS. Portanto é necessário que se tornem mais claros os processos legais envolvidos, bem como a forma com a qual os corpos serão preparados, conservados e utilizados em todas as áreas do conhecimento.

Frente a esta percepção, a disponibilidade de informações objetivas e claras sobre o processo de doação pode ter um reflexo na sociedade que permita a quebra de paradigmas que possibilite o aumento na taxa de adesão de possíveis doadores. Portanto, estratégias de comunicação que atinjam de forma mais efetiva a população, sobretudo a comunidade acadêmica, são essenciais. Tais medidas podem incluir a divulgação por meio de programas de rádio e televisão, internet, centros acadêmicos, cartazes afixados em murais de aviso das escolas, associações de bairros e centros comunitários.

Com base nestes resultados, novas pesquisas elucidando métodos que possam esclarecer as principais dúvidas ou inseguranças de possíveis doadores devem ser encorajadas, considerando a grande importância do contato com peças anatômicas naturais durante a formação de profissionais da saúde.

\section{Referências}

Abbasi, Asl J., Nikzad, H., Taherian, A., Atlasi, M. A., Naderian, H., Mousavi, G., Kashani, M. M., \& Omidi, A. (2017). Cultural acceptability and personal willingness of Iranian students toward cadaveric donation. Anatomical sciences education, 10(2), 120-126. 10.1002/ase.1634

Cintra, R. B. (2017). Desafios do Ensino da Anatomia Humana em Faculdades de Medicina. Revista Científica UMC, 2(1), 2525-5250.

Colares, M. A. M., de Melo, J. M., Vidotti, A. P. \& Sant'Ana, D. M. G. (2019). Metodologias de ensino de anatomia humana: estratégias para diminuir as dificuldades e proporcionar um melhor processo de ensino-aprendizagem. Arquivos do Mudi, 23(3), 140-160. 10.4025/arqmudi.v23i3.51527

Cordeiro, R. G., \& Menezes, R. F. (2019). A Falta de Cadáveres para Ensino e Pesquisa. Revista Brasileira de Educação Médica, 43(1), 579-587. 10.1590/1981-5271v43suplemento1-20190217.ing

Costa, G. B. F. D., Costa, G. B. F. D., \& Lins, C. C. D. S. A. (2012). O Cadáver no ensino da anatomia humana: uma visão metodologia e bioética. Revista Brasileira de Educação Médica, 36(3), 369-373. 10.1590/S0100-55022012000500011

da Rocha, A. O., de Campos, D., Farina, M. A., Pacini, G. S., Girotto, M. C., \& Hilbig, A. (2017). Using body donor demographics to assist the implementation of donation programs in Brazil. Anatomical sciences education, 10(5), 475-486. 10.1002/ase.1687 
Research, Society and Development, v. 10, n. 2, e55110212881, 2021

(CC BY 4.0) | ISSN 2525-3409 | DOI: http://dx.doi.org/10.33448/rsd-v10i2.12881

da Silva Baptista, J., Scardua, A., de Oliveira, G. B., de Souza, R., Leite, C. E. S., \& Marega, P. (2012). A influência das políticas brasileiras de expansão universitária no ensino de anatomia humana. O ANATOMISTA, 15

Dembogurski, J. E., Azenha, M. V., Amaral, A. P. C., Zeni, R. F., Silva, E. G., Cohen, S. P., Rocha, A. O., \& Hilbig, A. (2011). Dados preliminares de um modelo de programa de doação de corpos: Programa de Doação de Corpos da UFCSPA. Revista AMRIGS, 55(1), 7-10.

Medeiros, A. R. C., Neto, E. J. S., Saraiva, M. G., Coutinho, L. A. S. A., Neto, A. C. M., Soares, R. S., Alencar, C. M. P., Vieira, I. G. N. \& Silva, E. C. (2013). Dissecação e Capacitação de Habilidades e Competências Gerais na Formação Médica. Revista Brasileira de Ciências da Saúde, 17(3), $247-252$. 10.4034/RBCS.2013.17.03.06

Melo, E. M., \& Pinheiro, J. T. (2010). Procedimentos legais e protocolos para utilização de cadáveres no ensino de anatomia em Pernambuco. Revista Brasileira de Educação Médica, 34(2), 315-323. 10.1590/S0100-55022010000200018

Mitchell, P. D., Boston, C., Chamberlain, A. T., Chaplin, S., Chauhan, V., Evans, J., Fowler, L., Powers, N., Walker, D., Webb, H. \& Witkin, A. (2011). The study of anatomy in England from 1700 to the early 20th Century. Journal of Anatomy, 219(2), 91-99. 10.1111/j.1469-7580.2011.01381.x

Mwachaka, P. M., Mandela, P. \& Saidi, H. (2016). Repeated exposure to dissection does not influence students' attitudes towards human body donation for anatomy teaching. Anatomy research international, 2016. 10.1155/2016/9251049

Pereira, A. S., Shitsuka, D. M., Parreira, F. J., \& Shitsuka, R. (2018). Metodologia da pesquisa científica.

Portugal, H. S. P., Palma, P. C. R., Fraga, R., Riccetto, C. L. Z., Rocha, S., \& Carias, L. (2011). Modelo pélvico sintético como uma ferramenta didática efetiva comparada à pelve cadavérica. Revista Brasileira de Educação Médica, 35(02), 502-506. 10.1590/\$0100-55022011000400009

Queiroz C. D. A. F. (2005). O uso de cadáveres humanos como instrumento na construção de conhecimento a partir de uma visão bioética. Goiás. Mestrado [Dissertação] - Universidade Católica de Goiás.

Quiroga-Garza, A., Reyes-Hernandez, C. G., Zarate-Garza, P. P., Esparza-Hernandez, C. N., Gutierrez-de la, O. J., de la Fuente-Villarreal, D., Elizondo-Omaña, R. E., \& Guzman-Lopez, S. (2017). Willingness toward organ and body donation among anatomy professors and students in Mexico. Anatomical sciences education, 10, 589-597. 10.1002/ase.1705

Rocha, A. O., Tormes, D. A., Lehmann, N., Schwab, R. S., \& Canto, R. T. (2013). The body donation program at the Federal University of Health Sciences of Porto Alegre: A successful experience in Brazil. Anatomical sciences education, 6, 199-204. 10.1002/ase.1335

Volanek, A. F., \& Rissi, R. (2019). Perspectiva da doação voluntária de corpos para utilização no ensino de anatomia: consciência social, disposição e fatores associados. Revista de Ciências Médicas, 28(2), 77-84. 10.24220/2318-0897v28n2a4566 